\title{
Outcomes of mandibular Kennedy Class I and II prosthetic rehabilitation - an observational study
}

SADJ November 2019, Vol. 74 No. 10 p549 - p555

J Chamoko', S Khan ${ }^{2}$

\section{SUMMARY}

Loss of teeth may have a negative impact on appearance, nutrition and function. Removable prostheses for mandibular distal extension areas have been associated with more negative outcomes than with toothbounded saddles.

\section{Aim}

To describe the outcomes of rehabilitation with Kennedy Class / and /I dentures five years after insertion.

\section{Methods}

Dental laboratory and patient records were accessed to identify patients fitted with mandibular distal extension dentures between January 2011 and June 2017 by the Oral Health Centre of the University of the Western Cape. Information on the prosthesis, oral health status and study outcomes was recorded and augmented by telephonically interviewing 30 patients, randomly selected from the initial sample.

\section{Results}

Observed outcomes included 'low frequency of use' and 'high patient dissatisfaction.' Most common were: remakes $(n=26)$, abutment tooth extractions $(n=12)$ and repairs $(n=9)$. A large proportion $(n=105)$ of the sample received no follow-up treatment. No statistically significant associations existed between the outcomes and the variables of age, gender, type of opposing dentition, number of recalls and denture base material used.

\section{Conclusion}

Most commonly reported oral health problem associated with wearing Kennedy Class I and II dentures was abutment tooth loss. Remakes and repairs were frequent outcomes.

\section{Author affiliations:}

1. Joanna Chamoko: BChD; MSc (Dent), University of the Western Cape, Tygerberg, South Africa.

ORCID Number: 0000-0003-2958-1606

2. Saadika Khan: BChD; PDD (Clin Dent); MSc (Dent); PhD (Com Health), Senior Lecturer/ Principal Dentist, University of the Western Cape, Tygerberg, South Africa.

ORCID Number: 0000-0001-6017-959X

Corresponding author: Saadika Khan

Senior Lecturer/ Principal Dentist, University of the Western Cape,

Tygerberg, South Africa.

Email: skhan@uwc.ac.za

Author contributions:

1. Joanna Chamoko: Principal Researcher and thesis completion $-70 \%$

2. Saadika Khan: Supervisor of thesis and writing paper - $30 \%$

\section{ACRONYMS \\ FPDPs: $\quad$ Fixed Partial Denture Prosthesis \\ RPDPs: Removable Partial Denture Prosthesis \\ RPI: I-Bar System \\ SDA: $\quad$ Shortened Dental Arch}

\section{Keywords}

Clinical Outcomes, Mandibular distal extension dentures, Abutment tooth loss, Denture replacement, Repair, Patient opinion.

\section{INTRODUCTION}

Tooth loss due to caries, periodontal diseases and trauma is sometimes unavoidable. ${ }^{1}$ The World Health Organization $(\mathrm{WHO})$ guidelines indicate that the highest prevalence of partial edentulism occurs between the ages $35-44$ years $^{2}$ and that $12.6 \%$ of that sector of the adult population was completely edentulous. ${ }^{2}$

However, according to the South Africa Demographic and Health Survey (SADHS), 23\% of adults aged between 35-44 years were completely edentulous. ${ }^{3}$ Many South Africans must therefore be partially edentulous. The patterns of tooth loss do vary amongst different populations, ${ }^{4}$ and various studies have attempted to investigate the link between tooth loss and the different socio-economic factors between communities. ${ }^{5-7}$

Whilst not all lost teeth need to be replaced, rehabilitation of tooth loss is related to enhancing functions such as mastication and speech and aesthetics and may therefore be important. ${ }^{2}$

From the clinician's point of view, prosthetic rehabilitation aims to improve the distribution of occlusal forces on the remaining teeth, maintain the stability of the dentition and increase masticatory performance. In contrast, the perception of the patient of prosthetic rehabilitation is centered on the improvement of aesthetics and mastication with minimal discomfort and disruption to oral functions. ${ }^{1}$

Rehabilitation of shortened or posteriorly reduced dental arches, though, is not always necessary as sufficient masticatory function can be achieved with 20 teeth, having 9-10 posterior occluding pairs of teeth. ${ }^{8}$ Indeed it has shown that patients have scant knowledge of the consequences of missing teeth. ${ }^{9}$ 
Oral rehabilitation in partial edentulism, however, is not just to correct problems such as impaired mastication, aesthetics and speech, but also addresses the decline of patient-assessed quality of life that accompanies tooth loss. The major determining factor for treatment is the location of the lost tooth or teeth. The literature has shown that partial edentulism is more common in the mandible than the maxilla.

Younger adults tend to present with Kennedy Class III and IV partial edentulism. This is attributed to the early loss of the first molars as these teeth erupt first (and therefore become exposed to possible disease factors) and to loss of anterior teeth due to the susceptibility of children to trauma that affect these teeth. ${ }^{5}$

As individuals get older and lose more teeth the Kennedy Class III extends into a Class I and Class II. The Kennedy Class I and II partial edentulism is more common in the mandible while Classes III and IV being more common in the maxilla. ${ }^{5}$

Removable partial denture prosthesis (RPDPs) placement is more common with Kennedy Classes | and II while rehabilitation of Classes III and IV is usually with a fixed partial denture prosthesis (FPDPs) and implantsupported prostheses, depending on patient factors such as preference and finances, and on the condition of remaining teeth, and supporting tissues. ${ }^{5}$

Clinicians are more often faced with challenges in providing adequate support, retention and stability when restoring the mandibular distal extension spaces using RPDPs. Individuals may present with severely resorbed ridges due to disuse, migration and mal-positioning of posterior teeth, lost inter-arch space due to overeruption of opposing natural teeth; and teeth that are unable to serve as abutments due to their poor periodontal condition or unfavorable position after drifting. , $^{1,4}$

These sequelae also occur with other classes of partial edentulism. The impact is often magnified when mandibular posterior teeth have been lost early and distal partial edentulism is of long-standing duration. ${ }^{10}$

The most important factors that influence the success of prosthetic rehabilitation of Kennedy Class I and II mandibular arches maybe categorized as follows: ${ }^{1,11-18}$

1. Mechanical factors: Fractures of the major and minor connectors, ${ }^{17}$ requiring retreatment of the free-end RPDPs.

2. Biological factors: The wearing of RPDPs may be associated with an increased risk of caries and periodontal disease. ${ }^{16}$ Retention of a RPD is through tooth and ridge coverage, predisposing the teeth to plaque accumulation and bacterial overgrowth, possibly but not always leading to caries and periodontal disease. ${ }^{16}$

Isidor and Budtz-Jorgensen (1990) recalled patients biannually for the first two years then annually for three years and recorded high plaque scores and gingivitis but, remarkably, with no significant changes in probing depths. ${ }^{18}$ Their study does highlight the positive influence of recall visits on the success of newly placed RPDP's. ${ }^{18}$

3. Patient factors: Ensuring patient satisfaction is as important as treatment planning in defining success with the use of a prosthesis. ${ }^{1,3}$ Patients who consider the discomfort of a dental prosthesis to outweigh the perceived benefits will not wear it, with negative consequences on success.

Thus, a dentist considering prosthetic rehabilitation is wise to ensure he or she addresses all concerns expressed by patients. It has been recorded, though, that dental practitioners may be limited by the patients' poor oral hygiene, chronic illness (like diabetes) adverse social habits (like smoking) and, by the implications of financial cost. ${ }^{17}$

4. Biomechanical factors: Restoration of Kennedy Class | and || partially edentulous mandibles with RPDPs has historically posed biomechanical challenges because they derive support from two different tissues. ${ }^{15} \mathrm{~A}$ mandibular distal extension denture is supported by the periodontal ligament via the teeth, through the action of the rest seat, and by the mucosal tissues of the residual ridges.

Variable degrees of displaceability occur between these two tissues. ${ }^{11,12,19,20}$ These will definitely impact negatively on the comfort of patients and their ability to wear these prostheses and eventually on treatment outcomes.

In an effort to counteract these challenges, certain measures in RPDP treatment have been developed and applied, with varying degrees of success, including: special impression techniques, alternate RPDP designs such as the mesial rest combined with a proximal plate and I-bar (RPI) system, shortened dental arch (SDA), use of precision-attachments and implant-supported dentures. ${ }^{11,12,19,21-23}$

The aim of this study was to assess outcomes of treatment with mandibular Kennedy Class I and II prosthetic rehabilitation during a period of 5-6 years after insertion, by inspecting the file records of patients and through telephonic interviews. The following objectives were addressed:

1. To determine a demographic analysis of the patients and the types of dentures constructed, and to track the clinical history of the dentures.

2. To assess the opinions of patients of their prosthetic rehabilitation through telephonic interviews.

\section{METHODS}

This was a retrospective observational study involving qualitative data collection methods including a telephonic interview section including open-ended questions to allowing some of the sample of patients to share their opinions. From the records of the Oral Health Centre at the University of the Western Cape (UWC), a 
convenience sample was selected of patients who had been fitted with posterior mandibular prostheses during the period January 2011-June 2017. A smaller sample of 30 patients, a subset of this initial sample, was telephonically interviewed using a questionnaire with openand closed-ended questions. Records accessed were:

a). Dental laboratory records: On these records were clearly documented the personal details of patients, the type of the prosthesis, which dental arch, whether it was an acrylic or cobalt-chrome RPDP and when it was delivered to the patient.

b). Patients' dental records: Data recorded included the patient's age and contact details, the design of the prosthesis (Hospital instructions are to include a design within the patient folder), any repairs and remakes of the prosthesis, the loss of abutment teeth, and any recorded patient opinions concerning the prosthesis.

c). Telephonic interviews with patients: The information obtained from the Hospital records pertaining to the outcomes of the prosthetic rehabilitation was complemented by conducting 15-minute telephonic interviews with a small sample of patients, a subset of the initial sample.

These individuals verbally answered 12 questions related to the treatment received. Follow-up questions were asked where necessary to clarify answers to open-ended questions. The interviews were recorded to ensure an accurate account of the patients' responses, which were then entered into the personal files.

Patient participation was voluntary and informed consent was obtained before administering the questionnaire, following the principles of the Declaration of Helsinki.24

The following patients were excluded from the sample: if they: were fully edentulous; had incomplete dental records, wore prostheses rehabilitating Kennedy Classes III and IV, rehabilitation of Kennedy Class I and Class II using FPDPs, overdentures (ODs) or implant-retained prostheses and patients with any prostheses fabricated and fitted in other public or private clinics.

Data collection involved the completion of Excel spreadsheets with the information gleaned from accessed records, and from the summaries of the recording of the responses to the questionnaires used for the telephonic interviews. Data analysis included computation of standard descriptive and comparative statistics.

Frequency calculations of demographic details, patient records and questionnaire responses were completed and one sample or two sample t-tests of significance were calculated to determine the outcome of any associations; the information was grouped to ascertain the distribution of variables amongst specified intervals and in order to make meaningful deductions. Data collection and analysis were completed using Excel and SPSS software.

\section{RESULTS}

Ethical clearance was obtained from the UWC Biomedical Ethics Committee (Registration Number: BM $16 / 7 / 25)$. All participants had at the outset of treatment signed a consent form meeting the requirements of the Declaration of Helsinki. ${ }^{24}$

According to the technical laboratory records, 335 lower RPDPs had been made during the period January 2011 to June 2017. Of these, 160 were lower partial acrylic and 175 were lower partial metal (cobalt-chrome) RPDPs.

Access was gained to 269 patient clinical files (66 patient records were not found, 19.7\%). These recorded a total of 217 mandibular partial dentures, and the occurrence and percentage prevalence of the different dentures are shown in Table 1. From amongst the total patient records accessed, 152 complete patient records were found for patients who had been supplied with either a Kennedy Class I or Class II mandibular RPDP. Kennedy Class I ( $n=95)$ was the most common, whilst only 57 Kennedy Class II dentures had been delivered during the study period.

\begin{tabular}{|c|c|c|}
\hline Kennedy Class & Number & Percentage \% \\
\hline Class I & 95 & 44 \\
\hline Class II & 57 & 26 \\
\hline Class III & 58 & 27 \\
\hline Class IV & 7 & 3 \\
\hline Total & 217 & 100 \\
\hline
\end{tabular}

From the compiled data the observed outcomes recorded were:

a). Recall: At the time of the study, most patients (69\%) had not returned to the Oral Health Centre for any follow-up treatment.

b). Remakes: Twenty-six remakes had been required (17\%), usually due to poor fit, to reports of pain and discomfort, or mechanical failures and loss of abutment teeth.

c). Extraction of abutment teeth: Recorded were 12 instances $(8 \%)$. The reasons for extractions, whether due to periodontal disease or caries, were not clear.

d). Repairs were not commonly required ( $n=9,6 \%$ ), but were due to loss of an abutment tooth and subsequent tooth addition, and also to midline fractures or fractured clasps.

These outcomes were all considered an indication of treatment failures. Most remakes or repairs had occurred within the first two years of denture delivery. Relines and restored abutments could not be analyzed as outcomes because of the minimum occurrence.

Only one prosthesis of the 152 RPDP Kennedy Class I and Class || sample included in this study had been relined and only one individual had an abutment tooth restored following denture delivery. 


\section{a). Age distribution and outcomes}

The most common outcome recorded for this cohort is the number of 'remakes' of RPDPs, especially for those patients in the age category 65-74 and 75 years and above. No remakes were reported for individuals aged 25-34 years (Figure 1).

It was found that all expected cell frequencies were not greater than five, resulting in a chi squared test being unsuitable. A Fisher's Exact Test for association was therefore conducted between age categories and reported failures or study outcomes. Only a rather moderate weak negative association was found between age categories and study outcomes $(\varphi=-0.298 ; p=0.119)$.

\section{b). Gender distribution and mandibular distal extension RPDPs}

Females constituted the greater proportion (72\%) of individuals who had been rehabilitated with either mandibular Kennedy Class I (72\%) or Class II (58\%) RPDPs. A moderately weak association was shown between gender and Kennedy Classification $(\varphi=0.1403$; $p=0.082)$.

More denture failures were recorded for the female participants than their male counterparts. The most common negative outcome in both gender groups was 'remakes' of dentures.

Figure 2 is an illustration of the distribution of study outcomes in relation to differing conditions in the opposing maxillary arch and shows that the most common outcome for patients with mandibular distal extension RPDP treatment is 'remakes'.

However, Fisher's exact test did not reveal a statistically significant association and therefore the nature of the opposing arch did not significantly affect the outcome of the prosthetic treatment.

\section{Type of denture material and outcomes}

Having the denture remade was the most common outcome for both types of denture materials used as denture bases as shown in Figure 3. The graph also shows that the majority of denture failures occurred with those made with cobalt-chrome denture base material.

In determining whether an association exists between denture material and Kennedy Classification a chi square test was carried out, for the expected cell frequencies were greater than five.

The result showed there was no significant association between denture material and Kennedy Classification $(x 2=0.0502 ; p=0.823)$. The type of denture material, therefore, did not significantly influence the resulting outcome.

From the quantitative analysis the most common observations were that a large majority of the patients did not return for follow-up treatment, the prosthesis was either remade or repaired or the patient lost an
Age category and type of failure

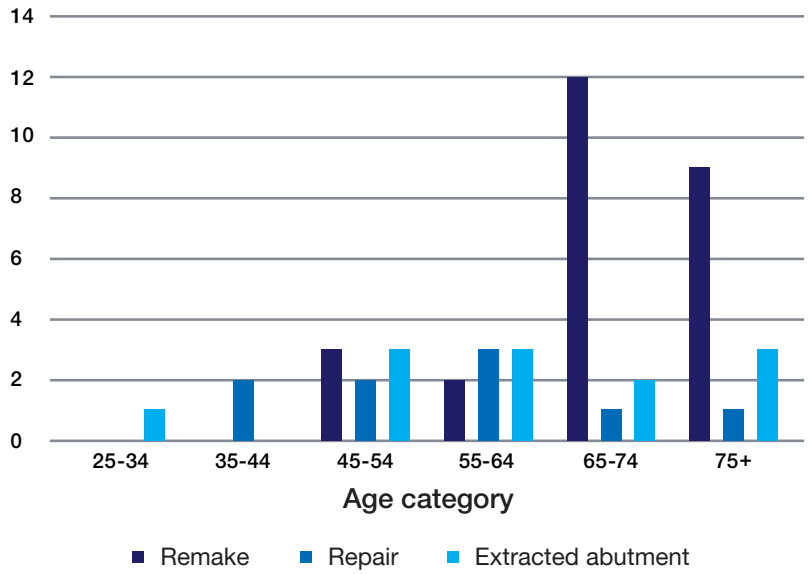

Figure 1. Graph indicating age distribution and study outcomes.

\section{Age category and type of failure}

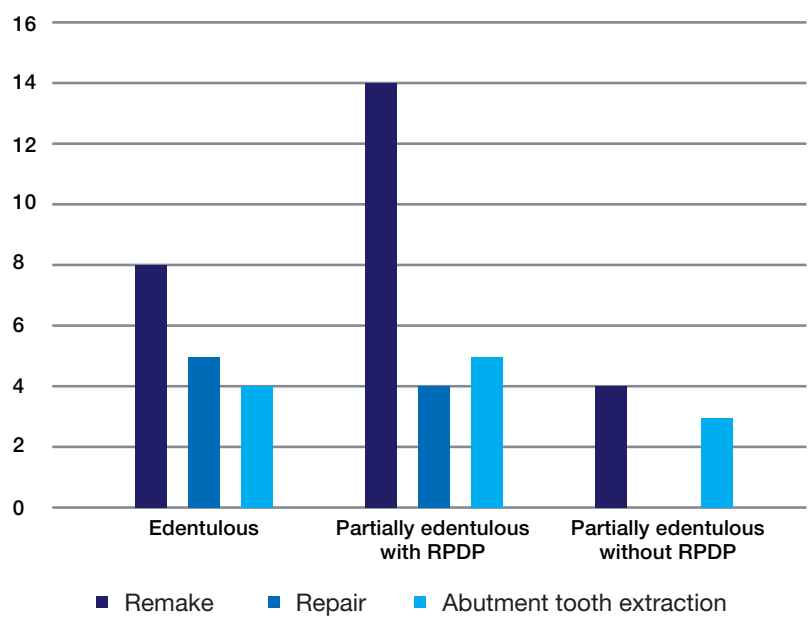

Figure 2. Frequency distribution of outcomes related to opposing dentition.

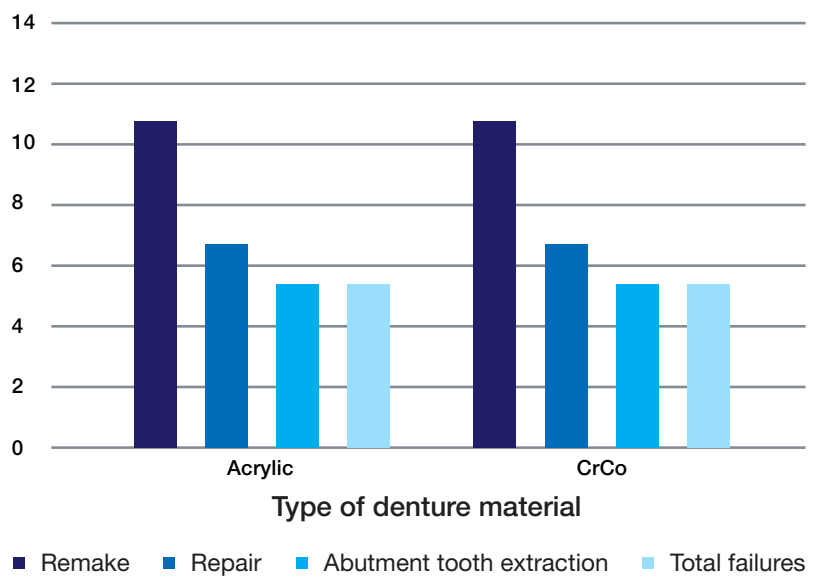

Figure 3. Graph indicating the two types of denture materials and study outcomes.

abutment tooth. About $27 \%$ of the remakes were prompted by the loss of an abutment tooth. Half of the repairs were tooth additions after the loss of an abutment tooth. 
There was, however, no statistical relationship between any of the outcomes including the loss of abutment teeth and the other measured variables. Since the remakes or repairs occurred within a short period of time (all occurred within two years of denture delivery) they are considered to be treatment failures as is the loss of abutment teeth.

The combined total number of these failures was 47 from amongst the sample of 152 (31\%). The remainder of the cases should not be considered as successes because when the sample of 30 individuals were interviewed, their responses made it clear that that was not always the case.

After consulting the literature, the following criteria were selected for further investigation through the telephonic interviews: the frequency and impact of wear, replacement and satisfaction with RPDPs.

Patient comments recorded with regards to the frequency and impact of wear indicated that most of them seldomly wore or did not wear the denture at all.

The reasons given were 'discomfort', 'painful', 'didn't fit properly' and 'can't eat or chew with it.' Their comments also centered on the position and poor aesthetics of the clasps necessary for retention, yet these were included according to the design and as per standard Oral Health Centre protocol.

The responses of patients regarding the replacement of dentures were hardly answered, and those who responded said 'they did not go back for another denture' or 'they were on a waiting list'.

Patients were requested to score their rate of satisfaction with dentures on a scale of 1 to 10. The low scores (mostly below 4) which 67\% of individuals gave confirmed their dissatisfaction with their RPDPs.

They recounted their experience of a negative impact on wearing of RPDP such as 'nothing improved with their dentures'. The contrary was obviously true for those minority of patients who reported wearing their 'denture all the time' or 'most of the time', as they were totally satisfied (with scores of 6 or more) and did not require replacement dentures. These individuals reported a positive impact on chewing and functioning.

All the RPDP patients wanted their appliances to ensure an improvement in their aesthetics and functioning, but many were clearly disappointed. They seemed unaware of the option of returning to the treatment center to have these denture problems corrected, which could have improved the denture experience (Many did return but for other reasons such as scaling and restorations).

\section{DISCUSSION}

The success of prosthetic rehabilitation is the shared responsibility between the clinician and the patient. ${ }^{25}$ This implies correct diagnosis, correct treatment planning and careful execution of the work together with patient education, the initial step in management and which continues throughout the treatment and maintenance stages.

Communication between the clinician and patients is key to successful treatment outcomes. The patient must understand the benefits and limitations of the treatment so that unattainable expectations are lowered and misuse of the prosthesis is prevented.

The patient also has a role to play in maintaining the oral tissues and the denture prosthesis through consistent hygiene practices. ${ }^{25}$ The literature has shown that RPDP wearers are prone to tooth loss as a result of periodontal breakdown and caries and the action of the denture as a Class I lever. ${ }^{11,13-16,19}$

The delivery of the denture does not signify the end of treatment but the patient is expected to attend follow-up visits to mitigate, at an early stage, any adverse effects of wearing the denture. ${ }^{14,16,26-27}$ The negative influence of RPDPs on oral health status can be minimized when a system of periodic recalls is implemented, studies showing a low incidence of caries, abutment tooth loss and periodontal disease. $3,14,16,26-27$

A significant proportion of the patients did not return to the treatment centre for monitoring. The outcome of loss of abutment teeth in this study could be attributed to multiple factors: poor oral hygiene practices by the patient, incorrect diagnosis, inadequate patient education about maintenance, poor selection of treatment options and improper denture design.

Patients showing poor adaptability to previous RPDPs may have benefited from fixed alternatives instead of multiple remakes. ${ }^{28}$ Certain individuals in the study population had their distal extension RPDPs remade three or four times in the period under observation.

The use of the RPI system ( $n=6)$ was limited in the study sample population and may indeed be regarded as outdated. The treatment choice based on the diagnosis and as it related to the problems observed was, therefore, poor. Satisfied patients, who scored the prosthetic treatment as 6 or higher, were pleased with how the denture improved their mastication. ${ }^{29}$ However, sixty seven percent of patients reported seldomly wearing the denture or not wearing it at all if discomfort or pain was experienced.

Some patients were functioning well with a complete maxillary prosthesis and the remaining anterior mandibular teeth. thus, a misdiagnosis of treatment could have been made as these patients may have been better suited for management with SDAs. ${ }^{22}$

Carr and Brown (2011) included the use of the altered cast technique as part of the six phases to providing a distal extension denture with the best support. ${ }^{25,27}$ The technique was not used at all to make the 152 prostheses observed during this study.

The outcome of loss of abutment teeth in this study could be attributed to multiple factors: poor oral hygiene 
practices by the patient, incorrect diagnosis, inadequate patient education about maintenance, poor selection of treatment options and improper denture design.

\section{Status of the prosthesis}

The study indicates that remakes and repairs are not only a parameter for measuring the status of the prosthesis but also of oral health and patient satisfaction, for remakes were prompted by three reasons: complaints about the fit of the denture, lost abutment teeth and fractures. ${ }^{26}$

\section{Patient opinion}

Koyama and colleagues (2010) carried out telephonic interviews of patients who had received an RPDP. ${ }^{30}$ Their criteria of determining successful frequency of wear were:

a). Successful: the original RPDP was worn daily for five years.

b). Remake: the original was replaced within five years.

c). Failure: the RPDP was not used or rather used sporadically.

The patients in the current telephonic study relayed the information that the most important patient factor in the success of treatment was the perceived benefit of the prosthesis and level of comfort. ${ }^{1}$ When these were not met, the denture was hardly or never worn. Most assessed their level of satisfaction as below 4, an indication of disappointment.

\section{CONCLUSION}

Within the limits of this study, it can be concluded that patients' expectations of rehabilitation with mandibular distal extension RPDPs are largely unmet and that they need to be educated in this regard not just about treatment prospects, but about alternatives such as overdentures, shortened dental arch and/or implant retained prostheses.

It can also be said that patient dissatisfaction with mandibular distal extension RPDPs is significant, though clinicians appear unaware of this. Patients are not informed of the need and of their right to return for further management or correction of treatment following the initial rehabilitation.

\section{Implications for practice}

Due to the high prevalence of partial edentulism in SA, it is crucial that successful rehabilitation with RPDPs must be enhanced, so that improved function, esthetics and satisfaction ensure successful prosthetic treatment outcomes. University teaching and clinical protocols should be revised to assist in overcoming the negative outcomes as reported with this study.

\section{Limitations}

This study relied on the records of patients who had received RPDPs and on telephone conversations with a limited number of patients. Had clinical examinations been conducted more precise information may have been gathered. Comparison of outcomes with patients wearing dentures other than those rehabilitating Kennedy Class I and II edentulous spaces would be instructive.

Account was not taken of the duration of time since fitting the denture, although it was noted that most repairs and remakes occurred within two years of denture delivery. More detailed investigation into the influence of different materials and of the opposing arch may have been warranted.

\section{References}

1. Preshaw P, Walls AWG, Jakubovics NS, Moynihan PJ, Jepson NJA, Loewy Z. Association of removable partial denture use with oral and systemic health. J Dent. 2011; 39 (11): 711-9.

2. Douglass C, Gammon M, Atwood D. Need and effective demand for prosthodontic treatment. J Prosthet Dent. 1988; 59 (1): 94-104.

3. Department of Health, Medical Research Council, OrcMarco. South Africa Demographic and Health Survey 2003. Pretoria, South Africa, 2007.

4. Jeyapalan V, Krishan CS. Partial edentulism and its correlation to age, gender, socio-economic status and incidence of various Kennedy's classes: A literature review. J Clin Diagnostic Res. 2015; 6(6): 14-7.

5. Budtz-Jorgensen E, Luan W, Holm-Pedersen P, Fejerskov O. Mandibular dysfunction related to dental, occlusal and prosthetic conditions in a selected elderly population. Gerodont. 1985; 1(1): 28-33.

6. Vadavadagi SV, Srinivasa H, Hajira N, Lahari M, Reddy GTP. Partial edentulism with socio-demographic variables amongst subjects attending dental teaching institutions. Indian J Oral Health. 2015; 7 (2); 60-3.

7. Prabhu N, Kumar S, D'souza M, Hegde V. Partial edentulousness in a rural population based on Kennedy's Classification: An epidemiological study. J Indian Prosthodont Soc. 2009; 9 (1): 18-23.

8. Gotfredsen K, Walls AW. What dentition assures oral function? Clin Oral Impl Res. 2007; 18(s3): 34-45.

9. Dosumo OO, Ogunrinde JT, Bamingbove SA. Knowledge of missing teeth in patients attending prosthetic clinic in $\mathrm{UCH}$ Ibadan. Annals of Ibadan Postgraduate Medicine 2014; 12(1): 42-8.

10. Applegate OC. The rationale of partial denture choice. $J$ Prosthet Dent. 1960; 10 (5): 891-908.

11. Ben-Ur Z, Shifma A, Aviv I, Gorfil C. Further aspects of design for distal extension removable partial dentures based on Kennedy classification. J Oral Rehabil. 1999; 26(2): 165-9.

12. Witter DJ, van Palenstein WH, Creugers NHJ, Kayser AF. The shortened dental arch concept and its implications for oral health care. Comm Dent Oral Epidemiol. 1999; 27: 249-58.

13. Wagner B, Kern M. Clinical evaluation of removable partial dentures 10 years after insertion: success rates, hygienic problems, and technical failures. Clin Oral Investig. 2000; 4(2): 74-80.

14. Jorge JH, Quishida CCC, Vergani CE, Machado AL, Pavarina AC, Giampaolo ET. Clinical evaluation of failures in removable partial dentures. J Oral Sci. 2012; 54(4): 337-42.

15. Dhingra K. Oral rehabilitation considerations for partially edentulous periodontal patients. J Prosthodont. 2012; 21 (6): 494-513.

16. Bergman B, Hugoson A, Olsson C. Caries, periodontal and prosthetic findings in patients with removable partial dentures: A ten-year longitudinal study. J Prosthet Dent. 1982; 48(5): 506-14. 
17. Budtz-Jørgensen E. Restoration of the partially edentulous mouth: A comparison of overdentures, removable partial dentures, fixed partial dentures and implant treatment. J Dent. 1996; 24(4): 237-44.

18. Isidor F, Budtz-Jørgensen E. Periodontal conditions following treatment with distally extending cantilever bridges or removable partial dentures in elderly patients: A five-year study. J Periodont. 1990; 61(1): 21-6.

19. 19. Krol AJ. Clasp design for extension-base removable partial dentures. J Prosthet Dent. 1973; 29(4): 408-15.

20. Owen CP. Fundamentals of Removable Partial Dentures. $2^{\text {nd }}$ Ed. Juta and Company Ltd. Johannesburg, South Africa; 2000.

21. Käyser A. Shortened dental arches and oral function. J Oral Rehabil. 1981; 8: 457-62.

22. Armellini $D$, von Fraunhofer $A$. The shortened dental arch: $A$ literature review. J Prosthet Dent. 2004; 92 (6): 531-5.

23. Khan SB, Omar R, Chikte UME. Perceptions regarding the shortened dental arch among dental practitioners in the Western Cape Province, South Africa. SADJ. 2012; 67: 61-8.

24. World Medical Organization. Declaration of Helsinki. Br Med J. 1996; 313: 1448-9.

25. Carr AB, Brown DT. McCracken's Removable Partial Prosthodontics. $12^{\text {th }}$ Ed. Elsevier. Missouri, USA. 2011; 8-15.

26. Vermeulen AHBM, Keltjens HMAM, van't Hof MA, Kayser AF. Ten-year evaluation of removable partial dentures: Survival rates based on retreatment, not wearing and replacement. J Prosthet Dent. 1996; 76: 267-72.

27. Chandler JA, Brudvik JS. Clinical evaluation of patients eight to nine years after placement of removable partial dentures. J Prosthet Dent. 1984; 51(6): 736-43.

28. Sunnegardh-Groneberg K, Davidson T, Gynther G, et al. A treatment of adult patients with partial edentulism: A systematic review. Int J Prosthodont. 2012; 25 (6): 568-81.

29. Wostmann B, Budtz-Jorgensen E, Jepson N, et al. Indications of removable partial dentures: A literature review. Int J Prosthod. 2005; 18(2): 139-45.

30. Koyama S, Sasaki K, Yokoyama M, Sasaki T, Hanawa S. Evaluation of factors affecting the continuing use and patient satisfaction with removable partial dentures over 5 years. J Prosthodont Res. 2010; 54: 97-101. 